\title{
Sensitivity of dynamic methods for damage detection in structural concrete bridges
}

\author{
M. Breccolotti ${ }^{\mathrm{a}, *}$, G. Franceschini ${ }^{\mathrm{b}}$ and A.L. Materazzi ${ }^{\mathrm{a}}$ \\ ${ }^{a}$ Department of Civil and Environmental Engineering, University of Perugia, Perugia, Italy \\ ${ }^{\mathrm{b}}$ Department of Industrial Engineering, University of Perugia, Perugia, Italy
}

\begin{abstract}
A critical analysis of the available methods for the assessment of structural concrete bridges through the observation of their dynamic response is carried out. A mathematical model for the evaluation of the natural frequencies shift due to bridge damage is applied to the case of an existing reinforced concrete bridge. The numerical results, along with the general structure of the mathematical problem, allow discussing some limitation and drawbacks of the dynamic methods. Namely the need for a reference set of the bridges dynamic properties before the onset of damage and the effect of the ambient temperature variation may lead, in many cases, to unreliable estimates of the structural condition of the investigated bridges.
\end{abstract}

Keywords: Bridge engineering, dynamic tests, structural concrete, damage assessment

\section{Introduction}

The management of road networks is a complex task that involves technical, economic and environmental aspects. A relevant issue is preserving the properties of bridges, maintaining them at the quality and performance level expected by the community. Bridges, in fact, are an important part of the property of a public institution and so the economic resources needed to maintain them efficient and safe must be allocated properly.

Road bridges are exposed, during their service lives, to the combined effects of aggressive environmental actions and mechanical damage due to vehicular traffic which can cause structural degradation.

Therefore they continuously ask for maintenance and rehabilitation works, to make them able to accommodate the vehicle traffic that becomes more and more fast and severe. Sometimes adjustments to new safety standards, new environmental issues and new needs of service are also needed.

Organized systems for the management of bridges are already operative in the USA and in Canada, while in Europe the EU funded specific research projects, like the "BRIdge Management in Europe - BRIME" [7].

Software packages are also available to manage the activities of inventory, inspection and maintenance of bridges, such as PONTIS [7]. They allow the agencies owning the bridges to carefully record inventory and inspection report practically without limits. The codes can foresee the deterioration progress of every bridge based on agency experience and external environment characteristics. According to the economical situation foreseen for the next years they allow also to allocate scarce resource in the best way to maintain bridges network functionality and safety at acceptable levels.

However the evaluation of bridges is largely based on the acquisition of data from visual inspections carried out periodically to control concrete deterioration and steel corrosion and to check and survey crack patterns.

This type of control can be affected by some shortcomings. First of all only visible defects can be revealed. Furthermore the evaluations are based on individual judgment which can vary from one operator to another one.

\footnotetext{
*Corresponding author. E-mail: breccolotti@strutture.unipg.it.
} 
To overcome these problems researchers are trying to develop objective methods of assessment based on the observation of bridge dynamic properties.

The application of these methods is unfortunately not straightforward, as some issues can affect the results of the dynamic tests.

Also the late Prof. Bruno Piombo, whose memory the special issue of Shock and Vibration containing this paper is dedicated to, dealt with this subject in 1995, in the wider frame of a research on the application of experimental modal analysis to bridge dynamics $[13,21,22]$.

The present study is a contribution to the discussion on advantages and drawbacks of the use of dynamic tests for the assessment of road bridges, paying special attention to the relevant case of structural concrete bridges.

\section{Dynamic tests in the diagnosis of bridges}

Since early 60 's the scientific community has directed his attention to the detection of the structural damage through the identification of changes in the dynamic parameters (such as eigenfrequencies, eigenvectors and damping). A wide literature survey about these methods, paying particular attention to bridge structures, was carried out by Doebling et al. in 1996. Current methods and techniques available for the damage prognosis, not only in civil engineering structures, are listed in Farrar et al. [12]. Different opinions emerged about the effectiveness of dynamic methods for damage identification. The oldest papers asserted that changes in vibration frequencies are good estimators of structural damage [19] while, subsequently, other studies demonstrated that frequencies changes alone are poor damage indicators [25].

Modal shapes seemed to be, in several cases, much more useful to determine and localize the damage. However Salawu et al. in 1994 compared the results obtained from several tests based on changes of modal shapes, asserting that relative variations of modal shapes are not good damage indicators when applied to experimental data.

Moreover some researchers underlined the fact that damage, to be detectable by means of dynamic methods, should be so relevant that in same cases it could cause the collapse of the structure even for small vehicular load [12, 25].

Farrar and Doebling in 1997 described the experiences relative to the dynamic tests carried out on the I-40 bridge over the Rio Grande at Albuquerque (NM), which was artificially damaged before its destruction. The results showed that the classical dynamic parameters (such as eigenfrequencies, eigenvectors and damping) were not enough sensible to damage but other algorithms, based on the increase of the structural flexibility near the damage location, seemed to be able to detect and localize the damage. The Authors, in hind sight, underlined also the importance of environmental factors, such as changes in temperature, traffic loading, wind speed, excitation method etc., which can affect the quality of the experimental measures.

In 2000 Maeck et al. described two methods for the determination of the stiffness decrease of structural concrete beams due to damage. The first method uses an updating algorithm based on the stiffness characteristics which depends, according to some hypothesis, on structural damage and on the comparison between the undamaged and the damaged structural response. This method uses only eigenfrequencies and is, therefore, impossible to distinguish asymmetric damage in a symmetric structure. The second method, called direct stiffness calculation, is able to detect and localize damage through modal shape determination. Other methods can be used to determine the presence and the location of damage.

Alampalli et al. in 1995 used standard modal analysis methods, like the Modal Assurance Criterion (MAC) and the Coordinate Modal Assurance Criterion (COMAC) during repeated tests on a simply supported steel and concrete composite beam. The bridge was artificially damaged with saw cuts on the steel beams. The Authors believed that the variations on eigenfrequencies could indicate the existence of the damage but the variations on modal shape were not enough sensible to indicate the position of the damage.

The same techniques were also used by Frýba and Pirner in 2001 to evaluate damage from the comparison between undamaged and damaged structural response. In particular the COMAC method was used with success by the Authors to assess the efficiency of repair works in a three span prestressed concrete segmental bridge. The dynamic behavior of the undamaged bridges was determined from a twin bridge located near the damaged one, which was taken as reference. 


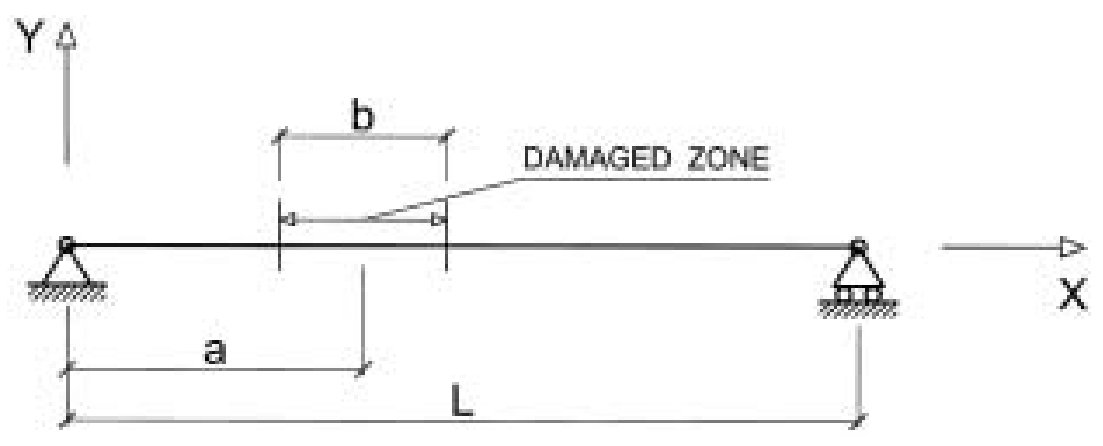

Fig. 1. Sketch of the model of the simple beam.

Brincker et al. [3] reported their experience in 15 progressive damage tests, artificially realized on the Z-24 bridge in Switzerland, razed and replaced by a new bridge. The technique used to determine the damage, called Enhanced Frequency Domain Decomposition (FDD), was based on the determination of eigenfrequencies, eigenvectors and damping changes. The Authors, neglecting the influence of environmental factor such as temperature on modal properties, believe that they can determine the presence of damage even for small changes of modal parameters.

In 2002 Ebert and Bucher investigated the influence of stochastic material properties on the damage process of crack evolution to show the importance of this effect on dynamic testing. The results of their experimental tests showed that the influence of stochastic material properties affect quite heavily the dynamic properties of the 20 equal reinforced concrete beams used in their investigation.

$\mathrm{Hu}$ and al. in 2002 used the Matched Damage Processing technique to determine presence, position and amount of damage in bridge structures. The algorithm compares the real structural response, acquired with traditional acquisition system, with the corresponding one, computed by means of the finite element technique and mode superposition. Some simulations realized on a simply supported beam, proved this technique to be able to correctly determine even little damage values, but it requires the knowledge of the undamaged dynamic behavior.

Anyway all the Authors agree on the fact that the damage detection in bridge structures using dynamic methods is a difficult task. Many parameters are involved in the process and often the variation of the dynamic properties behavior may be so small to be confused with the effect of changes in other non-mechanical parameters, like ambient temperature, moisture contents, the effect of non-structural masses added during the bridge service life, ice forming, boundaries condition changes, measurement errors and uncertainties always present when dealing with experimental data.

However, recent studies report promising results obtained by advanced techniques based on statistical analysis of the dynamic response, on suitable damage indicators, on neural networks and on model updating $[15,16,26,27]$.

\section{The use of natural frequencies in bridge damage evaluation}

All assessment methods are based on the observation of the changes of the bridge natural frequencies with respect to a known reference condition. A simple mathematical model can be used to establish a relation between the variation of the structural characteristics and the variation of the frequencies. Even if real bridge cases behave often as reinforced plate-like structures, simple supported beams and cantilever beams will be considered in this paper as representative of bridge structures.

\section{Simple beams}

Let us consider a simple supported reinforced concrete beam (Fig. 1). The structural damping and the temperature effects are neglected. As the dynamic excitation is supposed to produce low amplitude stresses, the structural behavior may be represented using a linear elastic model. The damage is supposed to be distributed over a unique 
portion of the beam of length $b$ and centered at a distance $a$ from the left hand support. It is represented by a suitable reduction of the flexural stiffness of the beam.

A first order perturbation procedure is applied to the eigenvalue problem. The eigenvalue equation for a beam of length L, without damping, can be solved through variables separation from the equation of the free vibration:

$$
\frac{d^{2}}{d x^{2}}\left[E J(x) \frac{d^{2} Y}{d x^{2}}\right]=\lambda m(x) Y
$$

where $Y(x)$ is the modal shape, $E$ is the modulus of elasticity, $J(x)$ the section moment of inertia, $m(x)$ is the mass per unit length and the purely imaginary eigenvalue, $\lambda$, is the square of the pulsation $\omega$.

The boundary conditions are those associated with simple bearings at the end of the beam:

$$
Y(0)=Y(L)=0, \frac{d^{2} Y}{d x^{2}}(0)=\frac{d^{2} Y}{d x^{2}}(L)=0
$$

A numerable infinity of eigenvalues, along with the corresponding modal shapes, can be obtained.

In the case of a prismatic beam, having constant cross section $(J(x)=J, m(x)=m)$ the pulsations are:

$$
\omega_{r}=r^{2} \pi^{2} \sqrt{E J / m L^{4}}
$$

and the corresponding normalized modal shapes are:

$$
Y_{r}(x)=\sqrt{2 / m L} \sin (r \pi x / L)
$$

In the general case, for the normalized modal shapes, we have:

$$
\int_{0}^{L} m(x) Y_{r}(x) Y_{s}(x) d x=\delta_{r s}
$$

and

$$
\int_{0}^{L} E J(x) \frac{d^{2} Y_{r}}{d x^{2}} \frac{d^{2} Y_{s}}{d x^{2}} d x=\lambda_{r} \delta_{r s}
$$

where $\delta_{r s}$ is the Kronecker delta which is 1 for $r=s$ and 0 for $r \neq s$.

The damage is modelled as a variation of the structural characteristics, by means of the decrement of the moment of inertia $\Delta J(x)$, without other alterations of the inertia characteristics; as a consequence the eigenvalues and the modal shapes undergo the variation $\Delta \lambda_{r}$ and $\Delta Y_{r}(x)$, respectively. It is also supposed that the damage does not affect the supports. Therefore the previously stated boundary conditions are still valid.

The eigenvalue equation for the damaged beam becomes:

$$
\frac{d^{2}}{d x^{2}}\left\{E[J(x)+\Delta J(x)] \frac{d^{2}\left[Y_{r}+\Delta Y_{r}\right]}{d x^{2}}\right\}=m(x)\left[\lambda_{r}+\Delta \lambda_{r}\right]\left[Y_{r}+\Delta Y_{r}\right]
$$

which can be developed as:

$$
\begin{aligned}
& \frac{d^{2}}{d x^{2}}\left\{E\left[J(x) \frac{d^{2} Y_{r}}{d x^{2}}+\Delta J(x) \frac{d^{2} Y_{r}}{d x^{2}}+J(x) \frac{d^{2} \Delta Y_{r}}{d x^{2}}+\Delta J(x) \frac{d^{2} \Delta Y_{r}}{d x^{2}}\right]\right\} \\
& =m(x)\left[\lambda_{r} Y_{r}+Y_{r} \Delta \lambda_{r}+\lambda_{r} \Delta Y_{r}+\Delta \lambda_{r} \Delta Y_{r}\right]
\end{aligned}
$$

Taking into account the Eq. (1) and neglecting the higher order terms, Eq. (8) becomes:

$$
\frac{d^{2}}{d x^{2}}\left\{E\left[\Delta J(x) \frac{d^{2} Y_{r}}{d x^{2}}+J(x) \frac{d^{2} \Delta Y_{r}}{d x^{2}}\right]\right\}=m(x)\left[Y_{r} \Delta \lambda_{r}+\lambda_{r} \Delta Y_{r}\right]
$$

Multiplying by $Y_{r}$, integrating over the whole length of the beam and considering the boundary conditions, Eq. (9) becomes:

$$
\begin{aligned}
& \int_{0}^{L} E \Delta J(x)\left(\frac{d^{2} Y_{r}}{d x^{2}}\right)^{2} d x+\int_{0}^{L} E J(x) \frac{d^{2} Y_{r}}{d x^{2}} \frac{d^{2} \Delta Y_{r}}{d x^{2}} d x \\
& =\lambda_{r} \int_{0}^{L} m(x) Y_{r}(x) \Delta Y_{r}(x) d x+\Delta \lambda_{r} \int_{0}^{L} m(x) Y_{r}(x)^{2} d x
\end{aligned}
$$


Using the normalization conditions of Eqs (5) and (6):

$$
\begin{aligned}
& \int_{0}^{L} m(x) Y_{r}(x) \Delta Y_{r}(x) d x=0 \\
& \int_{0}^{L} E J(x) \frac{d^{2} Y_{r}}{d x^{2}} \frac{d^{2} \Delta Y_{r}}{d x^{2}} d x=\frac{1}{2}\left(\Delta \lambda_{r}-\int_{0}^{L} E \Delta J(x)\left(\frac{d^{2} Y_{r}}{d x^{2}}\right)^{2} d x\right)
\end{aligned}
$$

the following expression can be obtained:

$$
\Delta \lambda_{r}=\int_{0}^{L} E \Delta J(x)\left(\frac{d^{2} Y_{r}}{d x^{2}}\right)^{2} d x
$$

Under the assumed hypotheses, Eq. (13) determines the increment of the r-th eigenvalue in relation to the decrement of the structural characteristics. It is obvious that the variation is more relevant for the eigenvalues associated with modal shapes displaying greater curvature around the damaged zones. The same result stands still for fixed-fixed beam or for fixed-pinned beams.

Assuming that the variation of the structural characteristics, $\Delta J(x)$, may be modeled as a constant decrement $\Delta J$ of the moment of inertia in a portion of beam of length $b$, centered in a point at $x=a$, Eq. (13) becomes:

$$
\Delta \lambda_{r}=\frac{2 E \Delta J}{m L}\left(\frac{r \pi}{L}\right)^{4} \int_{a-b / 2}^{a+b / 2}\left(\sin \frac{r \pi x}{L}\right)^{2} d x
$$

Taking into account that

$$
\Delta \lambda_{r}=2 \omega_{r} \Delta \omega_{r}
$$

the corresponding variation of the pulsation is:

$$
\Delta \omega_{r}=\frac{r^{2} \pi^{2} \Delta J}{L^{3}} \sqrt{\frac{E}{J m}}\left(\frac{b}{2}-\frac{L}{2 \pi r} \sin \frac{r \pi b}{L} \cos \frac{2 r \pi \alpha}{L}\right)
$$

and the relative variation of the pulsation is:

$$
\frac{\Delta \omega_{r}}{\omega_{r}}=\frac{1}{2 \pi r} \frac{\Delta J}{J}(\beta-\sin \beta \cos \alpha)
$$

where the dimensionless geometric parameters $\alpha=2 r \pi a / L$ and $\beta=r \pi b / L$ have been introduced. The Eq. (17) expresses the relative variation of the $\mathrm{r}$-th natural pulsation as the product of the relative variation of the structural characteristics by the function

$$
F(\alpha, \beta)=(\beta-\sin \beta \cos \alpha)
$$

that depends on $r$, on the damage's geometry and on the extent and position of the center of the damaged zone.

Since $F(\alpha, \beta)$ is always positive for $\beta>0$, the frequency's relative decrement corresponds to a decrement of the structural characteristics. If the center of the damaged zone is located at $\mathrm{L} / 4$ from the support, the relative frequency's decrement is proportional to the length of the damaged zone; for other positions of the damaged zone the deviation from the proportionality decreases for increasing values of $\mathrm{r}$, as the ratio $\sin \beta / \beta$ tends to zero.

The dependency of the relative variation of the $r$-th frequency on the structural geometry through the products $r a$ and $r b$ shows that, with the same relative variation of the structural characteristics, correlations can be found among the relative variations of different frequencies for determined geometries of the damage. As an example the variation of the r-th frequency turns out to be equal to that of the s-th frequency, if the relation $a_{s} b_{r}=a_{r} b_{s}$ among the geometric parameters is valid.

It is clear from the symmetry of the model, that is not possible to detect with Eq. (17) any difference of behavior if the center of the damage is located at $x=a$ or at $x=L-a$. From Eq. (17) can be derived also that, with the same damage, the same frequency variation can be found in all the cases in which $F(\alpha, \beta)$ is constant. This observation allows establishing a relation of equivalence between different damage conditions that can be useful in order to reduce the number of different cases, but shows the limits of a survey based only on frequencies, if the scope is to characterize not only the magnitude of damage, but also its position. 

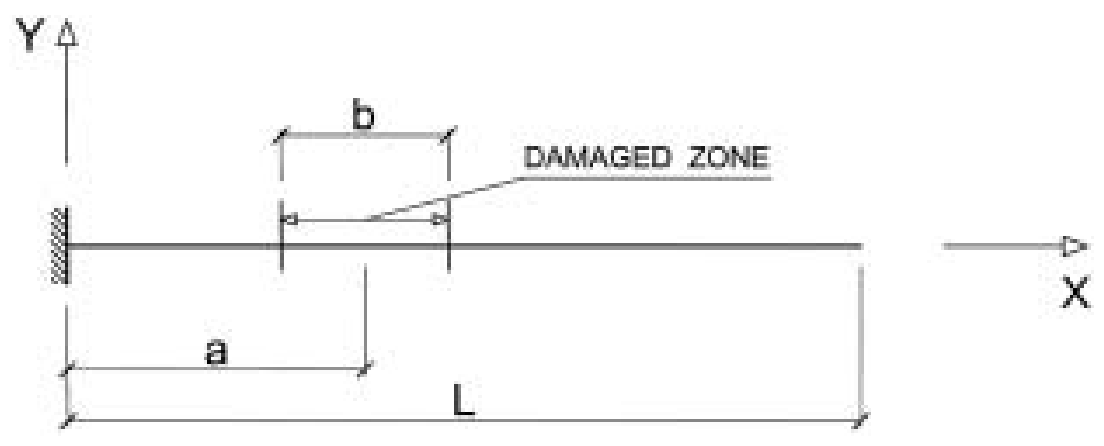

Fig. 2. Sketch of the model of the cantilever beam.

\section{Cantilever beams}

The result of Eq. (17) can be easily extended to the case of cantilever beams (Fig. 2).

The modal shapes are given by the following expression [4]:

$$
Y_{r}(x)=C \cdot\left[\cosh \left(\gamma_{r} x\right)-\cos (\gamma, x)-\frac{\cosh \left(\gamma_{r} L\right)+\cos \left(\gamma_{r} L\right)}{\sinh \left(\gamma_{r} L\right)+\sin \left(\gamma_{r} L\right)}\left(\sinh \left(\gamma_{r} x\right)+\sin \left(\gamma_{r} x\right)\right)\right]
$$

where $C$ is an arbitrary constant and $\gamma_{r} L$ are the roots of the equation:

$$
\cos (\gamma L) \cosh (\gamma L)=-1
$$

From this equation $\gamma_{r}$ is equal respectively to $1.8751 / \mathrm{L}, 4.6941 / \mathrm{L}, 7.8548 / \mathrm{L}, 10.9960 / \mathrm{L}, 14.1372 / \mathrm{L}$ and $17.2788 / \mathrm{L}$ for $r=1,2,3,4,5,6$.

The boundary conditions of the cantilever beam are:

$$
Y(0)=0, \frac{d Y}{d x}(0)=0, \frac{d^{2} Y}{d x^{2}}(L)=0, \frac{d^{3} Y}{d x^{3}}(L)=0
$$

Modeling the damage as already done for the simply supported beam, Eq. (13) becomes:

$$
\begin{aligned}
\Delta \lambda_{r}= & \int_{0}^{L} E \Delta J(x) \cdot C^{2} \cdot\left[r_{r}^{2} \cdot \cosh \left(\gamma_{r} x\right)+\gamma_{r}^{2} \cdot \cos \left(\gamma_{r} x\right)-\frac{\cosh \left(\gamma_{r} L\right)+\cos \left(\gamma_{r} L\right)}{\sinh \left(\gamma_{r} L\right)+\sin \left(\gamma_{r} L\right)}\left(\gamma_{r}^{2}\right.\right. \\
& \left.\left.\cdot \sinh \left(\gamma_{r} x\right)+\gamma_{r}^{2} \cdot \sin \left(\gamma_{r} x\right)\right)\right]^{2} d x \\
= & E \Delta J \cdot C^{2} \cdot \gamma_{r}^{4} \cdot \int_{a-b / 2}^{a+b / 2}\left[\cosh \left(\gamma_{r} x\right)+\cos \left(\gamma_{r} x\right)-\frac{\cosh \left(\gamma_{r} L\right)+\cos \left(\gamma_{r} L\right)}{\sinh \left(\gamma_{r} L\right)+\sin \left(\gamma_{r} L\right)}\right. \\
& \left.\left(\sinh \left(\gamma_{r} x\right)+\sin \left(\gamma_{r} x\right)\right)\right]^{2} d x
\end{aligned}
$$

The coefficient $C$ can be used to normalize the modal shapes with respect to the mass distribution.

By substituting Eqs. (19) and (5) becomes:

$$
m \cdot \int_{0}^{L} C^{2} \cdot\left[\cosh \left(\gamma_{r} x\right)-\cos \left(\gamma_{r} x\right)-\frac{\cosh \left(\gamma_{r} L\right)+\cos \left(\gamma_{r} L\right)}{\sinh \left(\gamma_{r} L\right)+\sin \left(\gamma_{r} L\right)}\left(\sinh \left(\gamma_{r} x\right)+\sin \left(\gamma_{r} x\right)\right)\right]^{2} d x=1
$$

For every modal shape the coefficient $C$ is equal to:

$$
C=\sqrt{\frac{1}{m \cdot 12}}
$$

The circular frequencies for a uniform cantilever beam are [4]: 


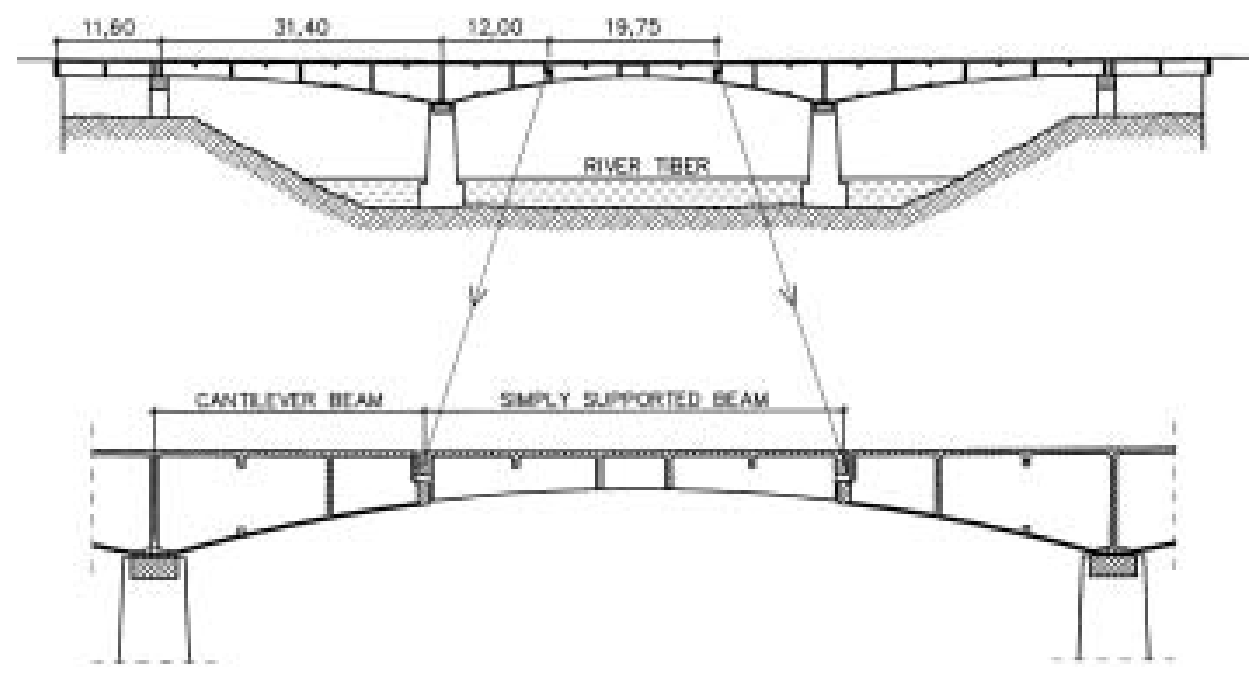

Fig. 3. Longitudinal cross section of the bridge.

$$
\omega_{r}=\left(\gamma_{r} L\right)^{2} \sqrt{\frac{E J}{m L^{4}}}
$$

Recalling Eq. (15), the variation in the pulsation is:

$$
\begin{aligned}
\Delta \omega_{r}= & \frac{L^{2}}{6(2 n-1)^{2} \pi^{2}} \sqrt{\frac{E}{J m}} \cdot \Delta J \cdot \gamma_{r}^{4} \cdot \int_{a-b / 2}^{a+b / 2}\left[\cosh \left(\gamma_{r} x\right)+\cos \left(\gamma_{r} x\right)\right. \\
& \left.-\frac{\cosh \left(\gamma_{r} L\right)+\cos \left(\gamma_{r} L\right)}{\sinh \left(\gamma_{r} L\right)+\sin \left(\gamma_{r} L\right)}\left(\sinh \left(\gamma_{r} x\right)+\sin \left(\gamma_{r} x\right)\right)\right]^{2} d x
\end{aligned}
$$

and consequently:

$$
\begin{aligned}
\frac{\Delta \omega_{r}}{\omega_{r}}= & \frac{\Delta J \cdot \gamma_{r}^{2} \cdot L^{2}}{6 J(2 n-1)^{2} \pi^{2}} \cdot \int_{a-b / 2}^{a+b / 2}\left[\cosh \left(\gamma_{r} x\right)+\cos \left(\gamma_{r} x\right)\right. \\
& \left.-\frac{\cosh \left(\gamma_{r} L\right)+\cos \left(\gamma_{r} L\right)}{\sinh \left(\gamma_{r} L\right)+\sin \left(\gamma_{r} L\right)}\left(\sinh \left(\gamma_{r} x\right)+\sin \left(\gamma_{r} x\right)\right)\right]^{2} d x
\end{aligned}
$$

\section{Application to a case study}

The results of the previous chapter have been applied to a reinforced concrete bridge over the Tiber river, located near the town of Rieti (Italy) and built between 1948 and 1949. The simple supported internal span of the bridge, $19.75 \mathrm{~m}$ long, was considered (Fig. 3). The transverse cross-section is indicated in Fig. 4. The following structural parameters have been assumed:

Young's modulus of concrete $E=35 \mathrm{kN} / \mathrm{mm}^{2}$;

flexural moment of inertia $J=0.9948 \mathrm{~m}^{4}$;

mass density $\rho=3.165 \mathrm{t} / \mathrm{m}^{3}$;

cross-section area $A=3.3282 \mathrm{~m}^{2}$;

mass density per unit length $m=10533.7 \mathrm{Kg} / \mathrm{m}$.

The previous densities are "equivalent values", suitably increased to take into account the structural self weight and the weight of the superstructure (pavement, sidewalk, parapet, ...). The moment of inertia of the undamaged structure, $J_{\text {uncr }}$, has been calculated for the uncracked section, neglecting the presence of the rebars.

The effect of damage is represented by a decrease of the moment of inertia estimated following the classical linear analysis of RC sections which assumes as null the tensile strength of concrete and uses the homogenization 
Table 1

Simple supported beam: Eigenpulsation variations for $b=0.5 \mathrm{~m}(\operatorname{span} L=19.75 \mathrm{~m})$

\begin{tabular}{cccc}
\hline$\Delta \omega / \omega[\%]$ & \multicolumn{3}{c}{$b=0.5 \mathrm{~m}$} \\
\cline { 2 - 4 } Mode no. & $a=2.0 \mathrm{~m}$ & $a=4.0 \mathrm{~m}$ & $a=\mathrm{L} / 2$ \\
\hline 1 & $-0,070$ & $-0,253$ & -0.717 \\
2 & $-0,254$ & $-0,654$ & -0.002 \\
3 & $-0,476$ & $-0,636$ & -0.714 \\
4 & $-0,650$ & $-0,229$ & -0.006 \\
5 & $-0,707$ & $-0,010$ & -0.708 \\
6 & $-0,628$ & $-0,284$ & -0.013 \\
\hline
\end{tabular}

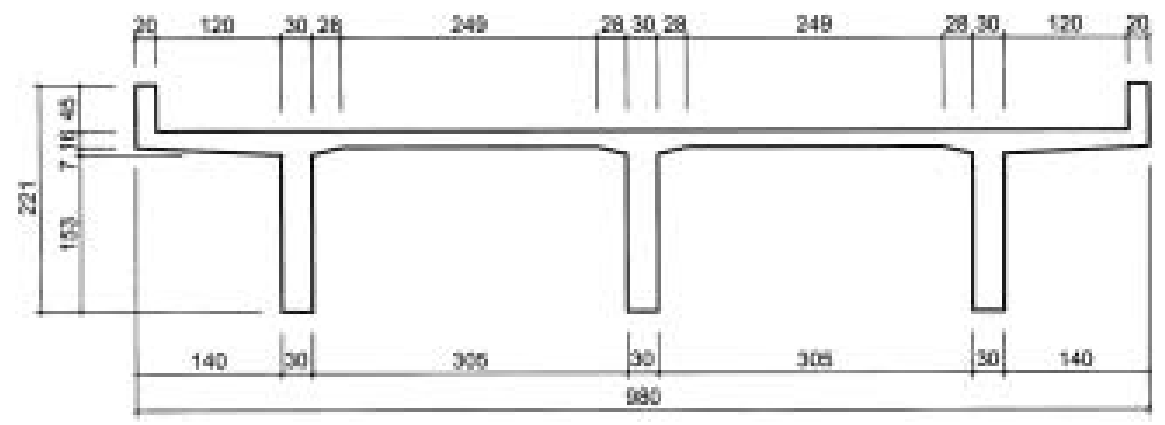

Fig. 4. Transverse cross section of the bridge.

technique. Under this hypothesis the moment of inertia reduces to $J_{d}=0.713 \mathrm{~m}^{4}$, with a decrement with respect to the undamaged section equal to approximately $28 \%$, figures which both depend on the geometric characteristics and on the steel content of the beam. When the damage extents along a finite portion of a beam, fully cracked sections are separated by uncracked regions and thus the average moment of inertia of the damaged part, $J$ ave, displays an intermediate value between $J_{d}$, which is a lower bound, and $J_{\text {uncr }}$, which is an upper bound. Several expressions are available in literature to estimate $J_{\text {ave }}$, like the one suggested by the ACI 318 (2002). However, in order to obtain an upper bound of the damage effect on the dynamic properties, $J_{\mathrm{d}}$ was used herein instead of the correct average value of the moment of inertia, $J_{\text {ave }}$.

Under this assumption, the first six pulsations of the undamaged structure may be found from Eq. (3):

$$
\begin{aligned}
& \omega_{1}=45.99 s^{-1} ; \omega_{2}=184.01 s^{-1} ; \\
& \omega_{3}=413.45 s^{-1} ; \omega_{4}=736.03 s^{-1} \\
& \omega_{5}=1150.05 s^{-1} ; \omega_{6}=1656.70 s^{-1}
\end{aligned}
$$

The application of the procedure previously outlined leads to the results of Tables 1 and 2 . The first one refers to a damage that extends for $b=0.5 \mathrm{~m}$ along the beam, at locations $a=2.0 ; 4.0$ and $\mathrm{L} / 2 \mathrm{~m}$. The second one refers to a damage extent of $b=2.0 \mathrm{~m}$. In the tables the relative variations of the first six natural frequencies for the various geometries of damage are showed.

The effect of damage is naturally greater in the case of Table 2 than in the case of Table 1 . In both cases the damage may be detected at the best when it is located at the midspan, using the first vibration mode. In the case of $b=0.5 \mathrm{~m}$ the maximum frequency shift is $-0.717 \%$, while in the case of $b=2.0 \mathrm{~m}$, the dynamic behavior is more damage sensitive, with a maximum frequency shift of $-2.845 \%$.

The analysis of the upper modes appears useful to detect damage located between the midspan and the supports: generally the frequency more affected by variation is the one which corresponding eigenvector displays its maximum value at the damage location.

In order to study the behavior of a cantilever beam, Eq. (27) has been applied to a structure 19.75/2 m long, having the same cross section and the same parameters as considered previously for the simple supported beam.

The relative variations of eigenfrequencies are summed up in Tables 3 and 4. 
Table 2

Simple supported beam: Eigenpulsation variations

for $b=2.0 \mathrm{~m}(\operatorname{span} L=19.75 \mathrm{~m})$

\begin{tabular}{cccc}
\hline$\Delta \omega / \omega[\%]$ & \multicolumn{3}{c}{$b=2.0 \mathrm{~m}$} \\
\cline { 2 - 4 } Mode no. & $a=2.0 \mathrm{~m}$ & $a=4.0 \mathrm{~m}$ & $a=\mathrm{L} / 2$ \\
\hline 1 & $-0,300$ & $-1,020$ & $-2,845$ \\
2 & $-1,041$ & $-2,542$ & $-0,095$ \\
3 & $-1,841$ & $-2,391$ & $-2,661$ \\
4 & $-2,326$ & $-1,037$ & $-0,357$ \\
5 & $-2,335$ & $-0,536$ & $-2,336$ \\
6 & $-1,987$ & $-1,280$ & $-0,725$ \\
\hline
\end{tabular}

Table 3

Cantilevered beam: Eigenpulsation variations for $b=$ $0.5 \mathrm{~m}$

\begin{tabular}{cccc}
\hline \multirow{2}{*}{$\begin{array}{c}\Delta \omega / \omega[\%] \\
\text { Mode no. }\end{array}$} & \multicolumn{3}{c}{$b=0.5 \mathrm{~m}$} \\
\cline { 2 - 4 } & $a=1.0 \mathrm{~m}$ & $a=2.0 \mathrm{~m}$ & $a=4.0 \mathrm{~m}$ \\
\hline 1 & -0.849 & -0.644 & -0.324 \\
2 & -0.397 & -0.054 & -0.194 \\
3 & -0.144 & -0.056 & -0.444 \\
4 & -0.028 & -0.304 & -0.039 \\
5 & -0.033 & -0.456 & -0.275 \\
6 & -0.119 & -0.357 & -0.488 \\
\hline
\end{tabular}

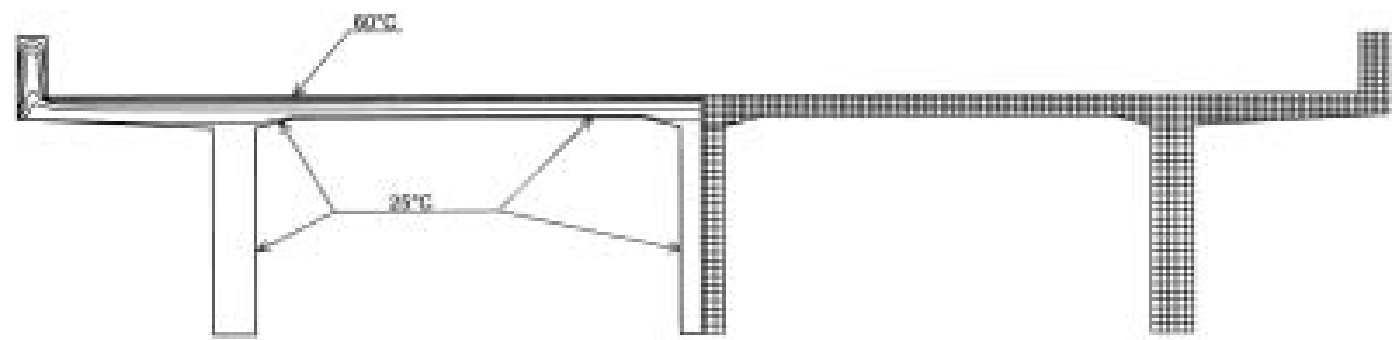

Fig. 5. Thermal map of the cross section after 240 minutes of exposure to heat.

In both cases of $b=0.5 \mathrm{~m}$ and $b=2.0 \mathrm{~m}$ the damage is detected at the best using the first vibration mode when it is located near the support or at $a=2.0 \mathrm{~m}$. In the case of $b=0.5 \mathrm{~m}$ the maximum frequency shift is $-0.849 \%$, while in the case of $b=2.0 \mathrm{~m}$, the maximum frequency shift is $-3.413 \%$.

Unlike the simple beams, the analysis of the upper modes appears to add little to the information of damage obtained through the use of the first mode.

\section{The role of the temperature on the dynamic field tests}

Both the actual boundary conditions and the temperature of the structural elements play an important role in the determination of the dynamic characteristics of bridges. A simple numerical simulation is carried out to evaluate the influence of the temperature on the stiffness properties of the structure.

The same bridge used in the sample analysis of the previous paragraph is supposed to have no damage. The relative variations of the eigenfrequencies as a consequence of temperature changes with respected to a reference value are computed.

As a realistic thermal condition, the upper surface of the deck is suppose to reach $60^{\circ} \mathrm{C}$, under the solar rays, while the bottom side of the bridge is supposed to be in contact with air at $25^{\circ} \mathrm{C}$. With this boundary conditions, supposing that the reference concrete temperature is $25^{\circ} \mathrm{C}$, and with the data supplied by the Eurocode 2 for the thermal properties of concrete, a thermal analysis is carried out by means of the FEM software FIRES T3 [18]. 
Table 4

Cantilevered beam: Eigenpulsation variations for $b=$

\begin{tabular}{cccc}
$2.0 \mathrm{~m}$ & \multicolumn{3}{c}{$b=2.0 \mathrm{~m}$} \\
\hline$\omega \omega / \omega[\%]$ & \multicolumn{3}{c}{} \\
\cline { 2 - 4 } Mode no. & $a=1.0 \mathrm{~m}$ & $a=2.0 \mathrm{~m}$ & $a=4.0 \mathrm{~m}$ \\
\hline 1 & -3.413 & -2.594 & -1.316 \\
2 & -1.804 & -0.412 & -0.807 \\
3 & -1.126 & -0.476 & -1.544 \\
4 & -1.002 & -1.068 & -0.549 \\
5 & -1.142 & -1.157 & -1.092 \\
6 & -1.256 & -0.956 & -1.177 \\
\hline
\end{tabular}

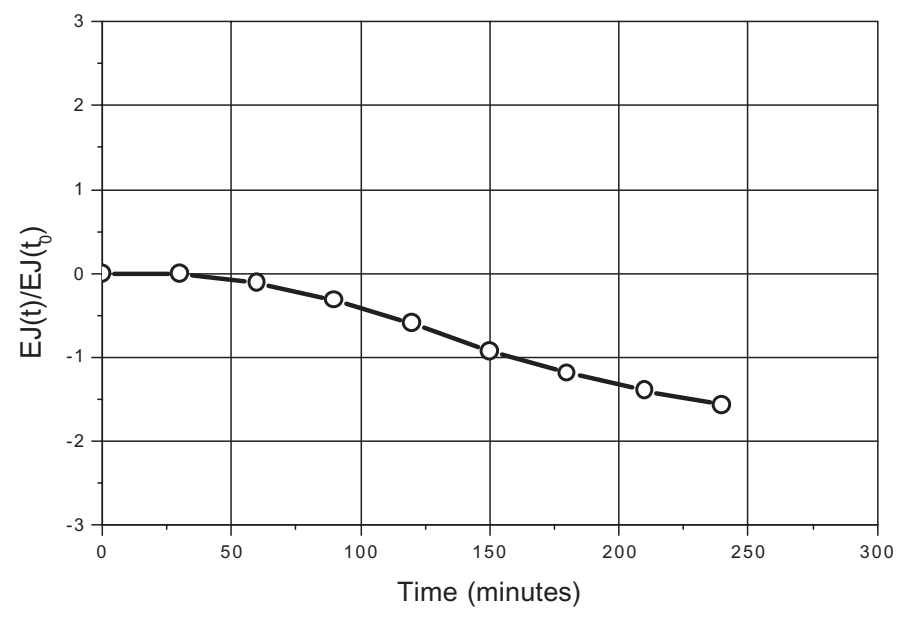

Fig. 6. Relative variation of the stiffness EJ with the time exposure to heat.

The analysis, under steady thermal boundary conditions, was carried out for a time duration of 240 minutes. In Fig. 5 is reported the thermal map of the cross section at the end of the computation.

As it is well known the Young's modulus of concrete varies with the temperature T.

The Model Code 90 issued by the C.E.B. in 1991 suggested the use of the following correlation:

$$
E(T)=E_{20^{\circ} \mathrm{C}} \cdot(1.06-0.003 \cdot T)
$$

Applying this relation, the stiffness EJ decreases while the temperature is growing.

The relative variation of the stiffness at time $t, E J(t)$, with respect to the initial stiffness $E J\left(t_{0}\right)$ versus time is showed in Fig. 6. It can be noted that after four hours of exposition ( $t=240$ minutes) the decrease of the global stiffness is greater than $1.5 \%$.

The frequency variation due to the variation of the stiffness $\Delta E J=E J(t)-E J\left(t_{0}\right)$ can be obtained differentiating Eq. (3) with respect to EJ, taken as a variable:

$$
\Delta \omega_{r}=\frac{r^{2} \pi^{2}}{\sqrt{m L^{4}}} \frac{\Delta E J}{2 \sqrt{E J}}
$$

Dividing Eq. (29) by Eq. (3), the ratio between the frequency variation and the frequency becomes:

$$
\frac{\Delta \omega_{r}}{\omega_{r}}=\frac{\Delta E J}{2 E J}
$$

Then, the decrease observed in the global stiffness $\Delta E J$ leads to a lessening of $0.75 \%$ in the pulsations of the beam.

This figure is of the same order of magnitude as most values reported on tables 1 to 4 for the damaged beams of the example. Thus temperature changes may shadow in many cases the frequency variations due to the mechanical damage. 


\section{Conclusions}

Dynamic methods for assessing structural concrete bridges are based on the observation of the structural response to a known dynamic input, on the modal analysis of data and on the comparison with a reference response. A first problem in this process is the knowledge of the dynamic properties of the investigated structure before the onset of damage. This information is not always available, because the dynamic inspections are not usually carried out at the end of the construction and because in many cases the mass and stiffness of bridges change in time due to modifications of the superstructure or refurbishing works. A viable solution could be to use FEM analysis to numerically compute the dynamic response of the undamaged structure, but this technique surely adds uncertainties to the whole assessment process.

The other major drawback of the procedure is the influence of ambient factors, like temperature variations, on the mechanical properties of the structural concrete, which change the structures dynamic response.

In the present paper a mathematical model which allows evaluating in closed form the shift of the natural frequencies with the location and the extent of damage has been proposed. The damage is modeled as a suited variation of the inertia properties.

A parametric analysis, during which the model has been applied to an existing reinforced concrete bridge, showed that the magnitude of the frequency shift barely reaches $3 \%$.

The effect of the variation of the ambient temperature on the dynamic response of bridges has been evaluated under reasonable hypotheses of the air temperature. The temperature-driven shift of the natural modes proved to be not negligible and sufficient to overshadow, in many cases, the effects of damage.

Therefore the dynamic methods for bridge monitoring and assessment based only on the detection of frequencies and modal shapes changes should be used with prudence. Other methods, such as those relying on pattern recognition, model updating, statistical analysis and neural networks, may be, instead, more efficient and reliable.

\section{References}

[1] American Concrete Institute (ACI) Building Code Requirements for Structural Concrete and Commentary, ACI 318-02/318R-02, 2002.

[2] S. Alampalli, G. Fu and E.W. Dillon, On the Use of Measured Vibration for Detecting Bridge Damage, Proc, Fourth International Bridge Engineering Conference, 1995, pp. 125-137.

[3] R. Brincker, P. Andersen and R. Cantieni, Identification and level I damage detection of the Z24 highway bridges, Experimental techniques, 2001.

[4] A.K. Chopra, Dynamic of Structures, Prentice-Hall, 1995.

[5] Comité Euro-International du Beton (CEB), CEB/FIP Model Code 90 (Final Draft), CEB Bulletin D’Information No. 203, 204, 205, Lausanne, Switzerland, 1991.

[6] Comité Européen de Normalisation (CEN), prENV 1992-1-2: Eurocode 2: Design of Concrete Structures. Part 1-2: Structural Fire Design, CEN/TC 250/SC 2, 2000.

[7] E. Czepiel, Bridge Management System: literature review and search, ITI Technical report n. 11, Northwestern University BIRL Industrial Research Laboratory, 1995.

[8] S.W. Doebling, C.R. Farrar, M.B. Prime and D.W. Shevitz, Damage identification and health monitoring of structural and mechanical system from changes in their vibrational characteristic: a literature review, Los Alamos National Laboratories, report LA-13070-MS, Los Alamos, New Mexico, 1996.

[9] M. Ebert and C. Bucher, Damage effects on the dynamic properties of $R / C$ beams - Experimental and numerical investigation, Proc. EURODYN-2002, 2002, pp. 1433-1438.

[10] C.R. Farrar and S.W. Doebling, Lessons learned from application of vibration-based damage identification methods to a large bridge structures, Proc. Intl. Workshop on Structural Health Monitoring, Stanford, CA, 1997, pp. 351-370.

[11] C.R. Farrar and K.M. Cone, Vibration testing of the I-40 bridge before and after the introduction of damage, Proceeding IMAC-1995, 1995, pp. 203-209.

[12] C.R. Farrar, H. Sohn, F.M. Hemez, M.C. Anderson, M.T. Bement, P.J. Cornwell, S.W. Doebling, J.F. Schultze, N. Lieven and A.N. Robertson, Damage prognosis: current status and future needs, Los Alamos National Laboratories, report LA-14051-MS, Los Alamos, New Mexico, 2003.

[13] A. Fasana, E. Giorcelli, B. Piombo and S. Sorrentino, Scaled model analysis of a traffic excited bridge, 1999 ASME Mechanics and materials conference, Blacksburg, 1999.

[14] L. Frýba and M. Pirner, Load test and modal analysis of bridges, Engineering Structures 23 (2001), $102-109$.

[15] L. Garibaldi, S. Marchesiello and E. Bonisoli, Identification and up-dating over the Z24 benchmark, Mechanical Systems and Signal Processing 17(1) (2003), 153-161.

[16] E. Görl and M. Link, Damage identification using changes of eigenfrequencies and mode shapes, Mechanical Systems and Signal Processing 17(1) (2003), 103-110. 
[17] S.-L.J. Hu, T.S. Hillier and P. Stepanishen, Data analysis and detection method for on-line health monitoring of bridge structures, University of Rhode Island, report n. URITC FY99-12, 2002.

[18] R. Iding, B. Bresler and Z. Nizamuddin, FIRES-T3. A computer program for the fire response of structures- Thermal, Fire Research Group, University of California. Berkeley, 1977.

[19] L.M. Lifshitz and A. Rotem, Determination of reinforcement unbonding of composites by a vibration technique, Journal of Composites Materials 3 (1969).

[20] J. Maeck, M. Abdel Wahab, B. Peeters, G. De Roeck, J. De Visscher, W.P. De Wilde, J.-M. Ndambi and J. Vantomme, Damage identification in reinforced concrete structures by dynamic stiffness determination, Engineering Structures 22 (2000), 1339-1349.

[21] S. Marchesiello, L. Garibaldi, B. Piombo and A. Fasana, Dynamics of multi-span continuous straigth bridges subjected to mdof moving vehicles excitation, Journal of Sound and vibrations 224(3), (1999), 541-561.

[22] B. Piombo, A. Fasana, S. Marchesiello and M. Ruzzene, Modelling and identification of the dynamic response of a supported bridge, Mechanical systems and signal processing 14(1) (2000), 75-89.

[23] A. Riva, L. Garibaldi and B. Piombo, Monitoring bridge framework: dynamic identification assessing structural damages, Proc. International Workshop on structural damage assessment using advanced signal processing procedures, Pescara, Italy, 1995.

[24] O.S. Salawu and C. Williams, Damage location using vibration mode shapes, Proceeding IMAC-1994, 1994, pp. $933-939$.

[25] C. Spyrakos, H.L. Chen, J. Stephens and V. Govidaraj, Evaluating Structural Deterioration Using Dynamic Response Characterization, Proc. Intelligent Structures, Elsevier Applied Science, 1990, pp. 137-154.

[26] A. Teughels, G. De Roeck, Damage assessment of the Z24 bridge by FE model updating, DAMAS 2003, 5th International Conference on Damage Assessment of structures, Southampton, UK, July 1-3, 2003, pp. 19-26.

[27] A. Teughels, J. Maeck and G. De Roeck, Damage assessment by FE model updating using damage functions, Computers and Structures 80(25) (2002), 1869-1879. 

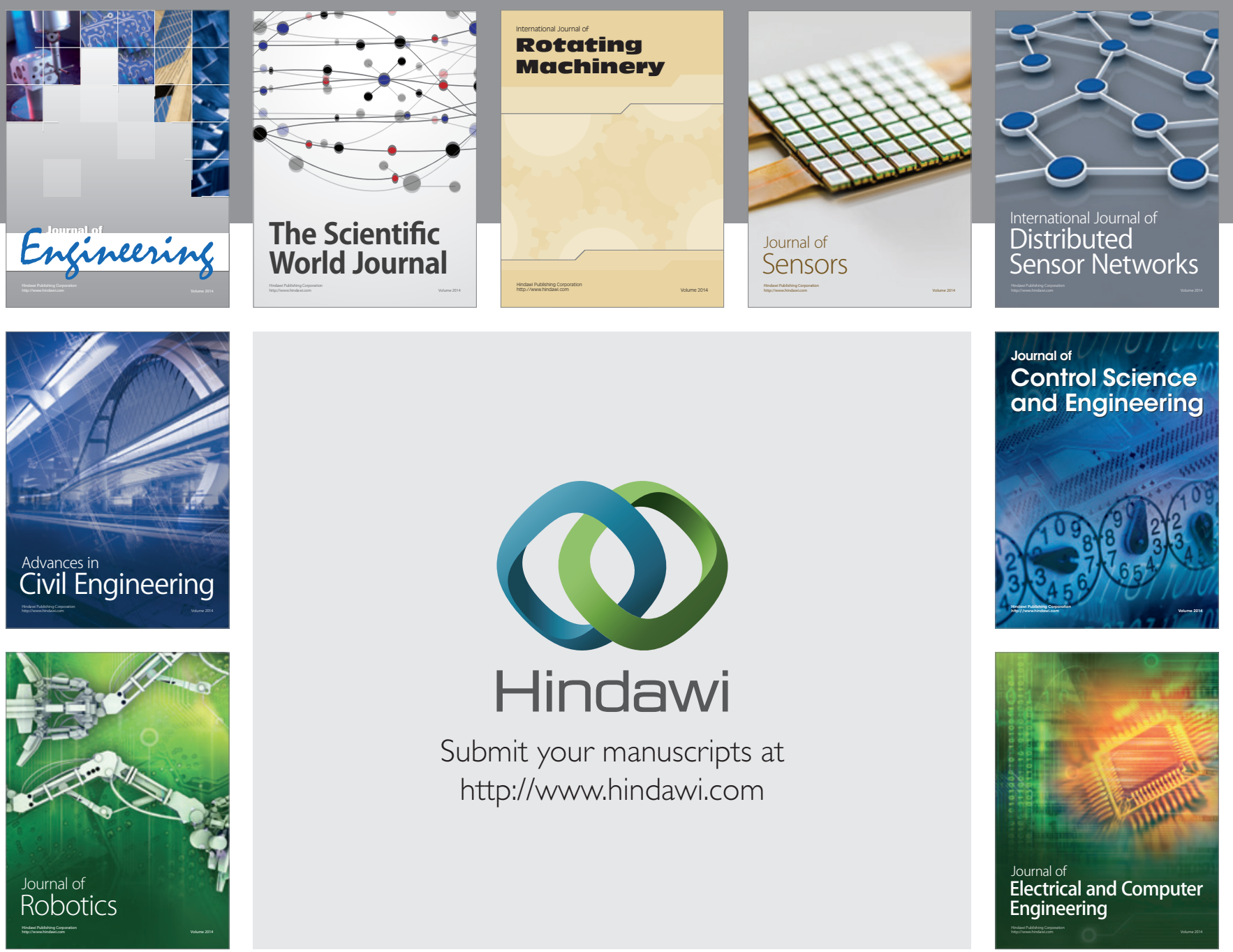

Submit your manuscripts at

http://www.hindawi.com
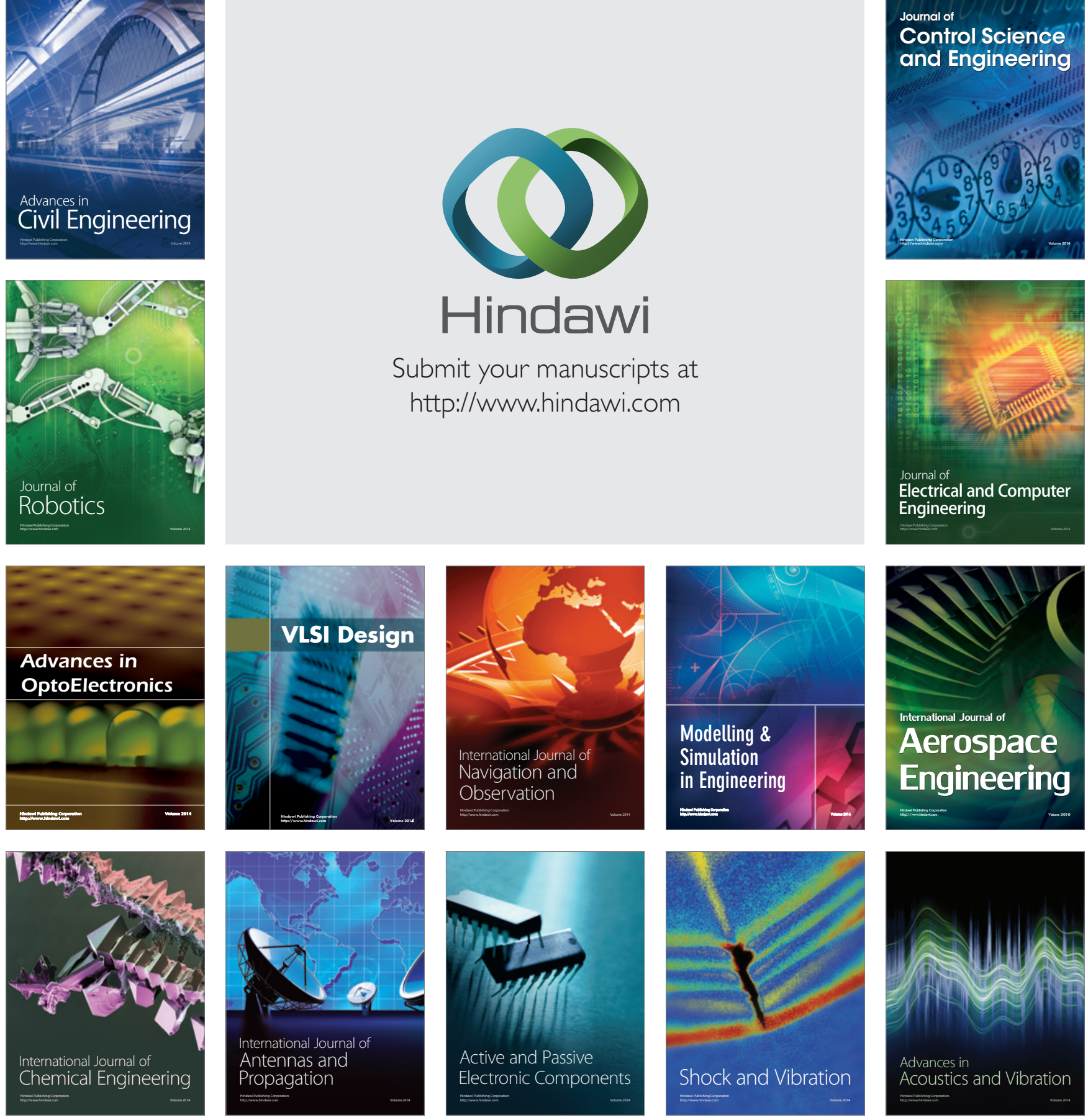